INPLASY

PROTOCOL

To cite: Zhang et al. Efficacy and safety of Banxia Xiexin Decoction as a complementary treatment for gastric cancer: A protocol for systematic review and meta-analysis. Inplasy protocol 202140060. doi: 10.37766/inplasy2021.4.0060

Received: 10 April 2021

Published: 10 April 2021

Corresponding author: Meiqing Zhang

miqi810305@163.com

Author Affiliation:

Bao'an Authentic Traditional Chinese Medicine Therapy Hospital

Support: At our own expense.

Review Stage at time of this submission: The review has not yet started.

Conflicts of interest:

None declared.

\section{Efficacy and safety of Banxia Xiexin Decoction as a complementary} treatment for gastric cancer: A protocol for systematic review and meta-analysis

Zhang, MQ1'; Huang, W2; Yuan, DK³.

Review question / Objective: Gastric cancer (GC) is one of the most common malignant tumors in the human digestive system, which seriously affects people's quality of life. As an effective treatment for GC, Traditional Chinese medicine (TCM) can effectively alleviate patients' clinical symptoms, improve the quality of life, and delay the life cycle. A large number of clinical studies have shown that Banxia Xiexin Decoction has shown a good effect in the treatment of GC. It has achieved good curative effect whether it is used alone or combined with radiotherapy and chemotherapy, which may play a more significant role in the treatment of GC. However, there is still no evidence of evidence-based medicine. Therefore, this study aims to systematically evaluate the efficacy and safety of Banxia Xiexin Decoction as a complementary treatment for GC.

INPLASY registration number: This protocol was registered with the International Platform of Registered Systematic Review and Meta-Analysis Protocols (INPLASY) on 10 April 2021 and was last updated on 10 April 2021 (registration number INPLASY202140060).

\section{INTRODUCTION}

Review question / Objective: Gastric cancer (GC) is one of the most common malignant tumors in the human digestive system, which seriously affects people's quality of life. As an effective treatment for GC, Traditional Chinese medicine (TCM) can effectively alleviate patients' clinical symptoms, improve the quality of life, and delay the life cycle. A large number of clinical studies have shown that Banxia Xiexin Decoction has shown a good effect in the treatment of GC. It has achieved good curative effect whether it is used alone or combined with radiotherapy and chemotherapy, which may play a more significant role in the treatment of GC. 
However, there is still no evidence of evidence-based medicine. Therefore, this study aims to systematically evaluate the efficacy and safety of Banxia Xiexin Decoction as a complementary treatment for GC.

Condition being studied: Gastric cancer(GC) refers to malignant tumors(MTs) derived from gastric mucosal epithelial cells, among which gastric adenocarcinoma is the most common, accounting for the leading cause of death of MTs in the world. According to the latest cancer statistics, there are more than $1,000,000$ new cases and 783,000 deaths of GC in the world in 2018, which is the fifthlargest incidence and the third-largest mortality of MTs. As one of the most common MTs in the human digestive system, GC is closely related to infection, diet, environment, and heredity. Because the onset of early GC is hidden, there are no clinical symptoms, and the popularity of gastroscopy screening is not high. About $90 \%$ of new cases have symptoms and often entered the advanced stage when undergoing clinical screening, which seriously affects patients' quality of life.At present, GC's main treatment methods include surgical resection, chemotherapy, molecular targeted therapy, and immunotherapy, but the prognosis of advanced GC is still not satisfactory due to factors such as easy recurrence, metastasis, and drug resistance.Therefore, it is essential for early treatment and prognosis of GC patients to explore the means with adjuvant treatment value actively. Traditional Chinese medicine(TCM) guides the prevention, diagnosis, and treatment of diseases with holistic concepts and treatment based on syndrome differentiation. As one of the important means to prevent and treat digestive system diseases, TCM has the characteristics of multiple targets and twoway regulation, which plays an important role in the treatment of GC with Western medicine, including regulating the immune function of the body, improving the tolerance of surgery and radiotherapy and chemotherapy, improving the preoperative physique, accelerating the postoperative functional recovery, alleviating the toxic and side effects of radiotherapy and chemotherapy, improving the clinical symptoms of patients, preventing and treating tumor recurrence and metastasis, improving the quality of life and delaying the life cycle. Banxia Xiexin Decoction comes from the classic book Treatise on Febrile Diseases by Zhang Zhongjing, a famous medical scientist in the Eastern Han Dynasty. It is widely used in the clinical treatment of various gastrointestinal diseases and has certain therapeutic effects on GC. Modern studies have also confirmed that Banxia Xiexin Decoction can not only inhibit the proliferation, invasion, and metastasis of GC cells by regulating the cell cycle of $G C$ and promoting the apoptosis of GC cells but also treat precancerous lesions of the stomach and relieve the gastrointestinal reaction of chemotherapy. Although many studies have shown that Banxia Xiexin Decoction, whether used alone or combined with radiotherapy and chemotherapy, has shown good effects in the treatment of GC, there is still a lack of large sample, multicenter, randomized, controlled and double-blind clinical evidence. Therefore, this study uses metaanalysis to evaluate the effectiveness and safety of Banxia Xiexin Decoction adjuvant therapy for GC in order to provide an evidence-based reference for clinical medication.

\section{METHODS}

Participant or population: Patients with advanced GC diagnosed by histopathology or clinical diagnosis will be included. Age, sex, course of the disease, source of case, region, and race are not limited.

Intervention: The treatment group was treated with Banxia Xiexin Decoction or Banxia Xiexin Decoction combined with routine treatment.

Comparator: The control group was given routine treatment, western medicine, or chemotherapy. 
Study designs to be included: We will collect all Randomized controlled trials (RCTs) of Banxia Xiexin Decoction as a complementary treatment for gastric cancer. Blind method, language is not limited.

Eligibility criteria: (1)Other TCMs were used in the control group; (2)Review, summarization, and repeated publications; (3)Research on incomplete data reporting, duplicate data, or inability to extract data; (4)Animal experimental literature; (5)Documents that do not meet the outcome indicators; (6)Complicated with other severe organs and systemic diseases; (7)No RCT.

Information sources: Two independent researchers searched the following English and Chinese databases: PubMed, Web of Science, MEDLINE, the Cochrane Library, Embase, China National Knowledge Infrastructure, the Chongqing VIP Chinese Science and Technology Periodical Database, Wanfang Database, and China Biomedical Database. The retrieval time of these databases is from the establishment of the database to March 2021. We will also manually search the Chinese Clinical Trial Register, Chinese Clinical Trial Register, conference papers, and other relevant literature.

Main outcome(s): This study's main outcome indicators are the effective rate of treatment(adopt the WHO general objective curative effect index of solid tumor) and the TCM syndrome score.

Quality assessment / Risk of bias analysis: Two researchers independently evaluated the bias risk included in the study and cross-checked the results. The randomized controlled trial bias risk assessment recommended by Cochrane Handbook is adopted for the bias risk assessment. The evaluation content mainly includes the following seven aspects: random sequence generation, Allocation hidden, Implement of the blind method for patients and testers, Implement the blind method for outcome assessors, The resulting data is incomplete, Selective reporting, Other biases. For each entry, "Yes" stands for "low bias risk," "No" stands for "high bias risk," and "unclear" stands for "unclear bias risk".

Strategy of data synthesis: Statistical analysis was carried out by RevMan 5.3 software. According to the heterogeneity included in the study, the correlation effect model was selected. If there is apparent clinical heterogeneity, sensitivity analysis or subgroup analysis can be used, or only descriptive analysis can be performed. Draw funnel charts for outcome indicators with more than ten studies, and analyze publication bias.

Subgroup analysis: If there is a heterogeneity source, we will make a subgroup analysis of this reason and then see if there is significant heterogeneity.

Sensitivity analysis: To judge the stability of the data synthesis, We will probably use sensitivity analysis for the data.

Country(ies) involved: China.

Keywords: gastric cancer, Banxia Xiexin Decoction, protocol, systematic review, meta-analysis.

Contributions of each author:

Author 1 - Meiqing Zhang Conceptualization, Data curation, Formal analysis, Investigation, Supervision, Writing - original draft, Writing - review \& editing.

Author 2 - Wei Huang - Conceptualization, Data curation, Investigation, Methodology, Software, Supervision, Writing - original draft.

Author 3 - Dongkai Yuan Conceptualization, Formal analysis, Investigation, Methodology, Software, Writing - review \& editing. 